\title{
Transfer of color matching in pigeons
}

\author{
RONALD L. MENLOVE \\ University of Delaware, Newark, Delaware 19711 \\ and \\ CHARLES P. SHIMP \\ University of Utah, Salt Lake City, Utah 84112
}

\begin{abstract}
Two pigeons were tested for transfer of matching to novel colors after a three-phase training procedure: First, the birds were trained to match colors; second, they were trained to choose odd colors; and third, they were retrained to match colors. The third phase included additional trials in which pecking at novel colors was reinforced. A subsequent transfer test showed that performance on trials with novel colors was slightly above chance but below levels observed on trials with only familiar colors. In addition, there was no difference between performance on trials in which both the sample and correct comparisons were novel and trials in which only the incorrect comparison was novel. These results provide some evidence for the acquisition of a general matching strategy, but they suggest that when pigeons are presented novel stimuli in a test for transfer of matching, their behavior displays biases that can lead to misinterpretations of what is learned in matching tasks.
\end{abstract}

In a matching-to-sample task, a subject is exposed to a sample stimulus and then to two comparison stimuli. A response to the comparison stimulus that is similar to the sample is reinforced, and a response to the comparison that is different from the sample is not reinforced. Several experiments designed to identify what pigeons learn in the matching problem have been based on a transfer-of-training paradigm (Cumming \& Berryman, 1965; Farthing \& Opuda, 1974). In this paradigm, subjects are trained to match with one set of stimuli. After a high level of matching performance is achieved, these stimuli are said to be familiar. Subsequently, a transfer test is administered with a novel set of stimuli. The results show that on transfer trials in which the sample and correct comparison are familiar and the incorrect comparison is novel, performance is as accurate as on trials in which all three stimuli are familiar. However, when the sample and correct comparison are novel, performance is no better than chance, and it is often significantly below chance. Both Cumming and Berryman (1965) and Farthing and Opuda (1974) have used results such as these to argue that pigeons do not attend to the incorrect comparison in matching-to-sample training. Rather, pigeons learn a conditional stimulus response chain in which a specific sample stimulus directs responses to a specific comparison stimulus.

However, there is an alternative description of transfer performance: On all trials in which the correct comparison is familiar, performance is good; on all trials in which the correct comparison is novel, performance is poor. Thus, pigeons may simply have a bias against pecking novel stimuli. This bias might mask the performance of whatever is learned in matching-to-sample training. The purpose of the present experiment was to evaluate this hypothesis. Here, we tried to neutralize this potential bias by reinforcing pecks at novel stimuli before those stimuli were used in the transfer test.

Previous experiments have investigated this hypothesis and have obtained contradictory results (Farthing \& Opuda, 1974). Subjects in only one out of four of Farthing and Opuda's experiments (Experiment 2) showed an effect of prior reinforced keypecks at the novel stimuli. However, these subjects had experienced several changes in the training stimuli: These subjects were first trained to match red, green, and blue colors, and then they were trained to match triangle, circle, and cross shapes. After two more changes between the color and shape dimensions, these subjects were trained to peck at yellow, and then they were tested for matching with yellow in the color set. These changes in the stimulus dimensions will be called nonreversal shifts in this paper. The subjects that did not show an effect of prior reinforced pecks at the novel stimuli were only trained with colors, trained to peck at yellow, and then tested for matching with yellow in the color set.

It is possible that the effects of nonreversal shifts and of reinforced pecks at novel stimuli combine to produce a difference between the results of Farthing and Opuda's (1974) Experiment 2 and those of Cumming and Berryman's (1965) experiment. That is, these effects might explain why the pigeons in Farthing and Opuda's experiment performed at equal levels with both types of novel stimuli, but the pigeons in Cumming and Berryman's experiment performed well when the incorrect comparison was novel but poorly when the sample and correct comparison were novel. The present experiment was designed to explore this possibility. Here subjects were first trained to match colors and then were 
trained to choose odd colors. This change in the task may be called a reversal shift. Finally, the subjects matched colors in a context involving interspersed trials in which pecks at novel colors were reinforced. Subsequently, a test for generalization of matching to the novel stimuli was administered.

\section{METHOD}

\section{Subjects}

Two White Carneaux pigeons were maintained at $80 \%$ of their free-feeding weights. Both pigeons had previous experience with multiple schedules using red and green colors. However, neither pigeon had been trained in a matching-to-sample problem.

\section{Apparatus}

Three Lehigh Valley three-key pigeon chambers were interfaced to a laboratory computer. Each key could be illuminated by an Industrial Electronics Engineers miniprojector with red, green, yellow, violet, or blue colors.

\section{Procedure}

The pigeons were first trained to match red, green, and yellow colors. At the start of each trial, the center (sample) key was lit with one of the three colors. A response on the sample key after $2 \mathrm{sec}$ illuminated the side (comparison) keys. One comparison key had the same color as the sample, and the second comparison key had a different color. The sample remained lit after the comparison keys were lit, but pecks on the sample key were no longer effective. A peck on the comparison key with the same color as the sample was followed by $1.5 \mathrm{sec}$ access to food; a peck on the comparison key with a different color was followed by a 1.5 -sec blackout. Each of the 12 possible combinations of the three colors was randomly presented on successive trials, and 120 trials were presented each day.

After 18 days of training with this procedure, the relation between the sample and comparison colors was changed from a matching to an oddity procedure. That is, after completion of the sample response requirement, a peck on the comparison key with a color different from the sample color was followed by $1.5 \mathrm{sec}$ access to food; a peck on the comparison key with the same color as the sample was followed by a $1.5-\mathrm{sec}$ blackout. Again, 120 trials were presented each day, and oddity training lasted for 45 days.

Following the oddity phase of training, the relation between the sample and comparison colors was changed back to a matching procedure. However, during the last training period, randomly interspersed between the matching trials were trials in which either violet or blue was displayed on one of the side keys. On these trials, only one key was lit, and a peck on the illuminated key was followed by $1.5 \mathrm{sec}$ access to food. These trials constituted $33 \%$ of all trials within these training sessions: On the average, 40 trials were novel color trials and 80 trials were matching trials. Because of limitations imposed by the displays in the miniprojector, only three different colors could be presented in any one session. Thus, the novel colors were introduced by substituting a single novel color for one of the familiar colors in a given session. As a consequence, 6 days were required to present the two novel colors in every combination with the three familiar colors. Both subjects were given 36 days of this training.

Finally, a generalization of matching was tested to the colors violet and blue, the colors to which pecks were recently reinforced, but which were novel with respect to the matching task. On a given transfer session, one of the novel colors replaced one of the familiar colors. Thus on Day 1 of transfer, violet replaced yellow, on Day 2, green, and on Day 3, red. On Day 4 blue replaced yellow, on Day 5, green, and on Day 6 , red. On each day the various possible color combinations were presented in random order, and 120 trials were presented in a session. Over the six transfer sessions, all combinations of the familiar and novel colors were presented, except violet was never presented with blue. A given set of color combinations appeared in only two of the six transfer sessions. For example, violet and yellow appeared together only in Transfer Sessions 2 and 3.

\section{RESULTS}

The means and standard deviations of the percentage of correct responses in transfer are shown in Table 1 . These numbers are based on the number of correct and incorrect responses to each color combination during the transfer test. The percentages were classified according to which of three types of stimuli were presented on a trial. The first classification separated trials with familiar or novel stimuli. Trials with novel stimuli were further classified according to whether the novel stimulus appeared as the sample and correct comparison or only as the incorrect comparison. Finally, means and standard deviations were obtained over each of the three stimulus classes for each bird.

The numbers in Table 1 can be combined to show that the percentage of correct responses was higher on trials with only familiar stimuli ( 86.5 and 92.0 for Birds 1 and 2, respectively) than on trials with novel stimuli (64.0 and 73.9 for Birds 1 and 2, respectively). However, there did not appear to be a consistent difference between trials in which the novel stimulus appeared as the sample and correct comparison and trials in which the novel stimulus appeared as the incorrect comparison.

The reliability of these observations was substantiated by contrasts in an analysis of variance on the combined data of both subjects. All statements of significance were made at the .05 level. Trials with only familiar stimuli were significantly different from trials with novel stimuli $[F(1,32)=14.7]$. The estimated percentage of variance due to this difference was 33 . However, trials in which the novel stimulus appeared as the sample and correct comparison were not significantly different from trials

Table 1

Means and Standard Deviations of the Percentage of Correct Responses in the Presence of Three Types of Transfer Stimuli

\begin{tabular}{|c|c|c|c|c|c|}
\hline \multirow[b]{3}{*}{ Bird } & \multirow{3}{*}{$\begin{array}{c}\text { Incorrect } \\
\text { Comparison }\end{array}$} & \multicolumn{4}{|c|}{ Sample and Correct Comparison } \\
\hline & & \multicolumn{2}{|c|}{ Familiar } & \multicolumn{2}{|c|}{ Novel } \\
\hline & & Mean & SD & Mean & SD \\
\hline 1 & $\begin{array}{l}\text { Familiar } \\
\text { Novel }\end{array}$ & $\begin{array}{l}86.5 \\
63.2\end{array}$ & $\begin{array}{l}6.4 \\
9.7\end{array}$ & 64.8 & 17.3 \\
\hline 2 & $\begin{array}{l}\text { Familiar } \\
\text { Novel }\end{array}$ & $\begin{array}{l}92.0 \\
71.5\end{array}$ & $\begin{array}{r}9.1 \\
26.7\end{array}$ & 76.2 & 16.8 \\
\hline
\end{tabular}

Note-Empty cells indicate that no trials involving only novel stimuli were used. 
in which the novel stimulus appeared as the incorrect comparison $[F(1,32)=.26]$. The estimated percentage of variance due to this difference was 4 .

The fact that performance on trials with novel stimuli was substantially worse than performance on trials with only familiar stimuli would indicate that the performance was not based entirely on a general same-different concept. However, the percentage of correct responses on trials with novel stimuli was significantly above .5 for each subject. This would indicate some transfer of color matching to novel stimuli.

Because the transfer test occurred over six sessions, it was possible for learning to occur over those sessions. This learning could account for the above-chance performance on trials with novel stimuli. To answer this argument, the data were further analyzed by separating Day 1 of exposure to a novel stimulus configuration from Day 2 of exposure to a novel stimulus configuration (recall that each stimulus configuration appeared in only two of the six transfer sessions). The percentage of correct responses was significantly above chance on the 1 st day that the novel stimuli appeared $\left(\chi^{2}=5.1\right.$ and 52.9 for Birds 1 and 2, respectively). Furthermore, a chi-square analysis conducted on the combined data of both subjects showed no significant difference between the 1st and 2nd day that a stimulus configuration appeared $\left(\chi^{2}=4.28\right)$. The estimated percentage of variance due to this difference was .4. Thus, the data suggest there was some transfer of color matching to the novel stimuli.

\section{DISCUSSION}

The present results replicate those in Experiment 2 of Farthing and Opuda (1974) for pigeons trained to match colors. Both experiments show no difference in performance between transfer trials in which the sample and correct comparison are novel and trials in which the incorrect comparison is novel. This result is strikingly different from traditional transfer-of-matching experiments, which show very good performance on transfer trials with novel incorrect comparison stimuli, but very poor performance on transfer trials with novel sample and correct comparison stimuli (Cumming \& Berryman, 1965). The pigeons in both the experiment by Farthing and Opuda and the present experiment were specifically rewarded for pecking at novel stimuli prior to transfer tests. The pigeons in Farthing and Opuda were also given successive nonreversal shifts with different stimulus dimensions prior to transfer. The pigeons in the present experiment were given successive matching-oddity reversal shifts prior to transfer. The extensive experiments of Farthing and Opuda provide convincing evidence that, by itself, reinforced pecking at novel stimuli will not produce a change in transfer performance. Thus, a combination of the specific training procedures in these experiments is required to produce the present transfer performance.

The present results are consistent with an argument that a bias against pecking at novel stimuli may affect transfer-ofmatching performance. This argument suggests that transfer performance is poor on trials in which the correct response is a peck at a novel stimulus; transfer performance is good on trials in which the correct response is a peck at a familiar stimulus. In the present experiment, the pigeons were rewarded for pecking at novel stimuli. Therefore, there were no completely novel stimuli; the transfer stimuli were only novel with respect to the matching task. This training should serve to reduce the bias against pecking at the transfer stimuli, and performance should have been the same on all novel stimulus trials. This was exactly the result obtained.

It is possible that the nonreversal and matching-oddity reversal shifts train the subjects to respond to the "meta-rules" of the matching task in much the same sense that reinforced pecks at novel stimuli train the subjects to respond to the novel stimuli. That is, without special training, a subject might respond to the transfer test as though it were an entirely new situation having no similarity to the previous matching training. In this new situation, the subject might respond with a position bias, a color bias, a win-stay lose-shift bias, and so on. However, nonreversal training with new stimulus dimensions or matchingoddity reversal training might serve to train the subject to respond to any new situation with a "general matching orientation." This general matching orientation could be described as extinction of any habit that would interfere with matching performance.

The results of the present experiment suggest the possibility that a pigeon's response to novel stimuli in transfer is affected by biases. These biases include avoidance of stimuli that have not been previously reinforced. Such biases can interfere with an experimenter's ability to determine what a subject learned in the original matching problem. These biases can be reduced by reinforcing pecks at novel stimuli and by successive reversals of matching and oddity tasks.

\section{REFERENCES}

Cumming, W. W., \& Berryman, R. The complex discriminated operant: Studies of matching-to-sample and related problems. In D. I. Mostofsky (Ed.), Stimulus generalization. Stanford, Calif: Stanford University Press, 1965.

Farthing, G. W., \& Opuda, M. J. Transfer of matching-tosample in pigeons. Journal of the Experimental Analysis of Behavior, 1974, 21, 199-213.

(Received for publication January 7, 1980.) 